\title{
Electron Collisions with Formic Acid Monomer and Dimer
}

\author{
Michael Allan \\ Department of Chemistry, University of Fribourg, Switzerland \\ (Received 22 November 2006; published 21 March 2007)
}

\begin{abstract}
Processes induced by the attachment of slow electrons to formic acid and its hydrogen-bonded dimer were studied. The elastic cross section and the cross section for the excitation of low quanta of discrete vibrations were found to be of a similar magnitude for both targets. A dramatic difference was found in the excitation of a vibrational quasicontinuum in the $1-2 \mathrm{eV}$ range with the ejection of very slow electrons $(E<0.1 \mathrm{eV})$, which was about $20 \times$ more intense in the dimer. The association of two formic acid molecules to form a dimer thus dramatically increases the power to quasithermalize electrons arriving with energies in the 1-2 eV energy range. Rapid electron-driven intracluster proton transfer is invoked to explain the observation.
\end{abstract}

The interest in electron-induced chemical processes has been recently renewed by the discovery that electrons at subionization [1] and even subexcitation energies [2] damage DNA. The biology-relevant discoveries inspired a series of gas phase studies of the various building blocks of DNA and other biomolecules [3,4]. The molecules in living tissue are, however, associated with neighboring molecules, often by means of hydrogen bridges, and it would thus be interesting to evaluate in what way this association affects the electron-induced processes.

The dimer of formic acid is a suitable model system for such study, because it is one of the most stable neutral complexes with a complexation energy of $\sim 15 \mathrm{kcal} \mathrm{mol}^{-1}$ $(0.65 \mathrm{eV})$. The present work characterizes the collisions by means of measurement of the cross sections for vibrational excitation and for elastic scattering. The cross sections provide a detailed insight into electron-driven processes and the role played by resonances (temporary negative ions). This method was recently applied to the formic acid monomer [5]. The information obtained in this way is complementary to that obtained by studying the dissociative electron attachment, recently performed on clusters of formic acid and on condensed formic acid [6-8], where a dramatic increase of cross sections with cluster size has been found.

Particularly important for the interpretation of the present observation is the discovery, by means of anion photoelectron spectra and quantum chemical calculations, that hydrogen-bonded dimer anions of carboxylic acids (including amino acids and formic acid) and nucleic acid bases have a proton transferred from the acid to the base in their most stable structures and that this structure is reached by a barrier-free proton transfer (BFPT) $[9,10]$. The BFPT structure was also predicted theoretically for the formic acid dimer anion [11].

Interesting phenomena were discovered in dissociative electron attachment to monomeric formic acid. The $\mathrm{HCOO}^{-}$formate anion signal appeared at its energetic threshold at $1.25 \mathrm{eV}$ [12]. The mechanism was clarified theoretically by Rescigno et al. [13]. High signal-to-noise ratio spectra revealed weak structures on the dissociative electron attachment band [12]. The absolute elastic cross sections as a function of scattering angle for energies between 1.8 and $50 \mathrm{eV}$ were measured by Vizcaino et al. [14] and compared to calculation by Gianturco and Lucchese [15].

The present measurements were performed under two experimental conditions. Under high backing pressure conditions, the liquid formic acid was kept at room temperature $\left(25^{\circ} \mathrm{C}\right)$, and the vapor in equilibrium with the liquid was introduced into the interaction region through a $30 \mu \mathrm{m}$ diameter nozzle without constricting the flow by a needle valve. The heat generated by the thermionic cathode raised the temperature of the nozzle to $35^{\circ} \mathrm{C}$. The vapor pressure at $25^{\circ} \mathrm{C}$ is $57.4 \mathrm{mbar}$ [16], and the molar fraction of the dimers at this pressure and at $35^{\circ} \mathrm{C}$ can be calculated using the equilibrium constant determined by Coolidge [17] to be $71 \%$. Under low backing pressure conditions, the pressure of the vapor was first reduced by a needle valve to 1 mbar (measured with a capacitance manometer) and then entered the interaction region through a $250 \mu \mathrm{m}$ diameter nozzle, also at $35^{\circ} \mathrm{C}$. The vapor had enough time to reach the equilibrium dimer concentration at $1 \mathrm{mbar}$ and $35^{\circ} \mathrm{C}$, which corresponds to a dimer molar fraction of $11 \%$.

The measurements were performed using a spectrometer with hemispherical analyzers [18]. All spectra were recorded at a scattering angle of $135^{\circ}$. The energy scales and the analyzer response function were determined as described in the earlier work on the monomer [5].

In the first stage of the experiment, electron energy-loss spectra were recorded both at the low and the high backing pressures and suitably subtracted to obtain spectra of the pure monomer and of the clusters shown in Fig. 1. The correct subtraction factors were determined using spectral ranges where only the monomer or only the dimer have vibrational frequencies. In particular, the dimer gives no signal at the free $\mathrm{O}-\mathrm{H}$ stretch frequency of $443 \mathrm{meV}$, and conversely the monomer has no frequency at $31 \mathrm{meV}$, the dimer in-plane rock vibration. The dimer spectrum was 


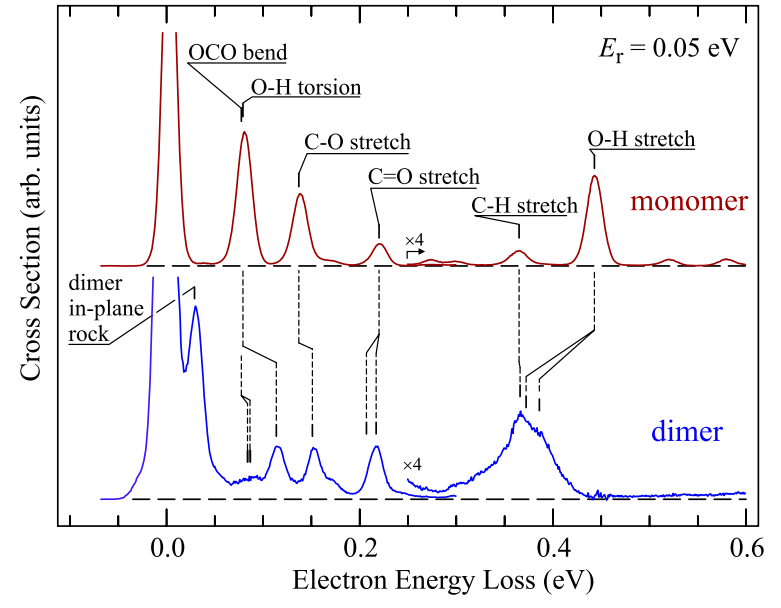

FIG. 1 (color online). Electron energy-loss spectra of the monomer and the dimer of formic acid.

thus obtained by subtracting the low-pressure spectrum from the high-pressure spectrum until the monomerspecific band at $443 \mathrm{meV}$ disappeared.

The energy-loss spectra in Fig. 1 were assigned by reference to the experimental vibrational frequencies which were listed and compared to calculations by Chang et al. [19] and Florio et al. [20]. The characteristic changes of the vibrations caused by the dimerization are borne out in the spectra. The $\mathrm{O}-\mathrm{H}$ stretch frequency is dramatically lowered and split into the $A_{g}$ and $B_{u}$ components. The $\mathrm{O}-\mathrm{C}=\mathrm{O}$ bend vibration is only slightly shifted and the $\mathrm{O}-\mathrm{H}$ torsion vibration more blueshifted, with very small splittings. The $\mathrm{C}-\mathrm{O}$ stretch vibration is slightly blueshifted, and the $\mathrm{C}=\mathrm{O}$ stretch vibration is slightly redshifted, whereby only the IR active higher frequency combination $B_{u}$ and not the lower frequency combination $A_{g}$ has appreciable intensity in the energy-loss spectrum under the conditions of near threshold excitation, as expected. The $\mathrm{C}-\mathrm{H}$ stretch frequency remained nearly unchanged. Nearly all of the signal observed in the lower spectrum can be thus assigned to the spectrum of the dimer, but the weak diffuse signal in the $0.32-0.36 \mathrm{eV}$ energy-loss range could stem from larger clusters or other structures of the dimer.

Insight into resonant processes is gained by recording the cross section for vibrational excitation as a function of electron energy as illustrated by the example of the $\mathrm{C}=\mathrm{O}$ stretch vibrations in Fig. 2. An enhancement of the cross section in the monomer reveals the ${ }^{2} A^{\prime \prime}$ shape resonance at $1.9 \mathrm{eV}$, resulting from a temporary occupation of a $\pi^{*}$ orbital [5]. It has already been observed in early electrontransmission studies [21,22], and the pronounced "boomerang" structure indicates a relatively narrow autodetachment width, less than $0.1 \mathrm{eV}$.

The monomer resonance is split into the ${ }^{2} A_{u}$ and ${ }^{2} B_{g}$ resonances in the dimer, with vertical attachment energies of 1.40 and $1.96 \mathrm{eV}$, respectively. No boomerang structure is observed in the dimer cross sections with the present

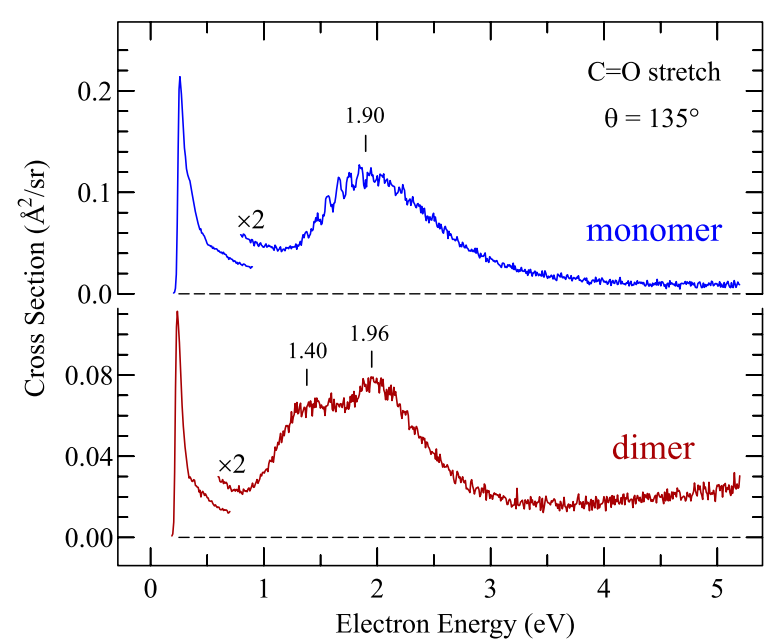

FIG. 2 (color online). Cross sections for the excitation of the $\mathrm{C}=\mathrm{O}$ stretch vibrations for the monomer and the dimer of formic acid.

level of statistical noise - the lack could be a consequence of a vibrational congestion due to a larger number of vibrational modes or could indicate a larger autodetachment width. The two shape resonances of the dimer were calculated at 2.87 and $3.68 \mathrm{eV}$, respectively, by Gianturco et al. [8] - this is higher than measured but within the expectation for scattering calculations with a simplified treatment of target polarization.

Threshold peaks such as seen in both cross sections in Fig. 2 have been observed in many polar (such as $\mathrm{HBr}$ ) and also some unpolar (such as $\mathrm{CO}_{2}$ ) molecules [23]. They are a consequence of dipole bound vibrational Feshbach resonances or of virtual states. The lower height of the threshold peak in the dimer is presumably the consequence of the lack of dipole moment in the equilibrium geometry of the dimer.

The subtraction factors required to obtain a pure dimer spectrum and determined as described above in connection with Fig. 1, together with the known molar fractions of the monomer and the dimer under the low- and high-pressure conditions and with the monomer absolute elastic cross section determined earlier [5], permitted the determination of the absolute cross sections for the dimer. The absolute elastic cross section of the monomer at $2 \mathrm{eV}$ and $135^{\circ}$ was measured to be $1.5 \AA^{2} / \mathrm{sr}$ [5]; that of the dimer is determined in this work to be $1.36 \AA^{2} / \mathrm{sr}$, with an estimated error of $\pm 40 \%$. It is surprising that the larger molecule has a lower cross section, although the $\pm 40 \%$ error bar of the dimer absolute value would also be compatible with a dimer cross section slightly larger than that of the monomer. But even with this uncertainty the measurement clearly indicates that the dimer cross section is substantially smaller than twice the monomer cross section. A permanent dipole moment generally increases elastic cross section at low energies, and the observation could thus be explained by the sizable dipole moment $(1.4 \mathrm{D})$ of the 
monomer and its absence in the dimer. The finding is also qualitatively compatible with the calculations [8], which predict the integral cross section at $2 \mathrm{eV}$ to be only very slightly larger for the dimer than for the monomer, and with the differential cross section calculations at 1 and $5 \mathrm{eV}$ [15], which allow the same conclusion.

Figure 3 shows a spectrum of scattered electrons obtained with an incident energy of $2 \mathrm{eV}$. The spectrum is plotted as a function of the energy loss - the elastically scattered electrons appear on the left; electrons leaving with nearly zero energy appear on the right. The spectra are similar as far as the elastic peak and the (selective) excitation of discrete vibrations in the energy-loss range up to about $0.6 \mathrm{eV}$ are concerned but differ dramatically in the height of the peak of very low energy electrons. The observation where electrons leave nearly all of their energy in the internal modes of a polyatomic target without exciting any specific vibrational modes (sometimes termed "unspecific excitation") was made already in the formic acid monomer [5] and in many other molecules [24]. The striking result reported here is that the association of two molecules to form a dimer dramatically enhances this phenomenon - the near zero $\mathrm{eV}$ peak is about $6.5 \times$ higher in the dimer in Fig. 3. It will be shown below that at $1.3 \mathrm{eV}$, where this effect peaks, the near zero $\mathrm{eV}$ peak is about $20 \times$ higher in the dimer than in the monomer.

Figure 4 shows the yield of the very slow electrons as a function of incident energy. The spectra were brought to

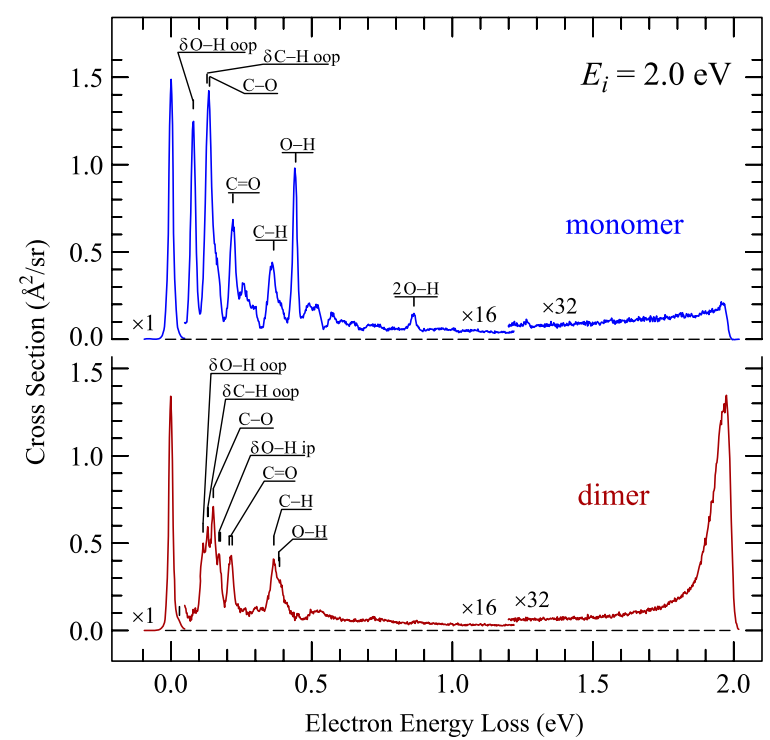

FIG. 3 (color online). Distribution of kinetic energies of electrons ejected after an impact of $2 \mathrm{eV}$ electrons on the formic acid dimer and monomer, plotted against the energy loss. The elastically scattered electrons have an energy loss of zero; a group of electrons having selectively excited specific vibrations is observed in the energy-loss range of $0.1-0.5 \mathrm{eV}$. A third group of electrons, with nearly zero kinetic energies, is observed around $2 \mathrm{eV}$ energy loss. The latter group is much more pronounced in the dimer than in the monomer. the same vertical scale by normalizing to the zero $\mathrm{eV}$ peaks ( $2 \mathrm{eV}$ energy loss) in Fig. 3. The two excitation mechanisms, one selectively exciting specific vibrations, the other populating a vibrational (quasi)continuum at larger energy losses, can be clearly distinguished. The quasicontinuum band in the monomer is clearly much weaker (it is shown expanded $4 \times$ ). It peaks at $1.65 \mathrm{eV}$, slightly lower than the peak in the vibrational excitation cross section in Fig. 2. The band peaks at $1.24 \mathrm{eV}$ in the dimer, slightly below the ${ }^{2} A_{u}$ shape resonance identified in Fig. 2. A way to formulate the observation is to say that the association of the formic acid by hydrogen bridges dramatically increases its power to quasithermalize electrons arriving in the 1$2 \mathrm{eV}$ energy range.

In search of the explanation for the dramatic difference between the monomer and the dimer, we notice the prediction of quantum chemical calculations that the attachment of an electron to formic acid dimer initiates a spontaneous proton transfer [11]. In the process of the reaction, the charge initially partially localizes on one of the formic acid molecules, where it is stabilized by distortion from planarity (driven by $\pi^{*} / \sigma^{*}$ vibronic coupling). The negatively charged formic acid is a strong base and attracts a proton, yielding a formate anion and a nonplanar dihydroxymethyl radical:<smiles>CC(C)OC(=O)O</smiles>

Hypothetical potential surfaces for the reaction are shown in Fig. 5. The initial resonant state of the anion formed by a vertical electron attachment is stabilized by symmetry lowering and a rapid proton transfer, thus quenching the fast autodetachment channel, which would otherwise dominate the scattering and lead to elastic scattering and selective excitation of low vibrational levels.

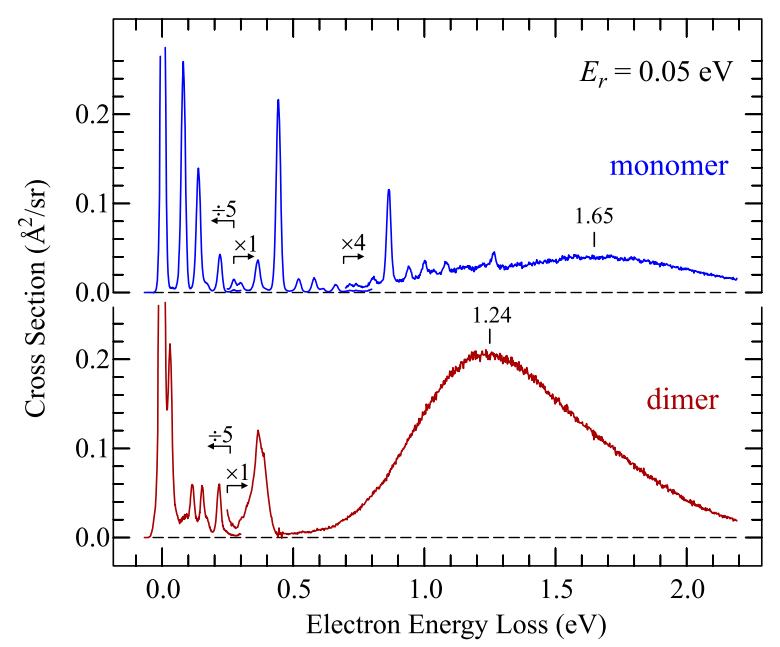

FIG. 4 (color online). The yield of very slow electrons recorded as a function of the incident electron energy. 


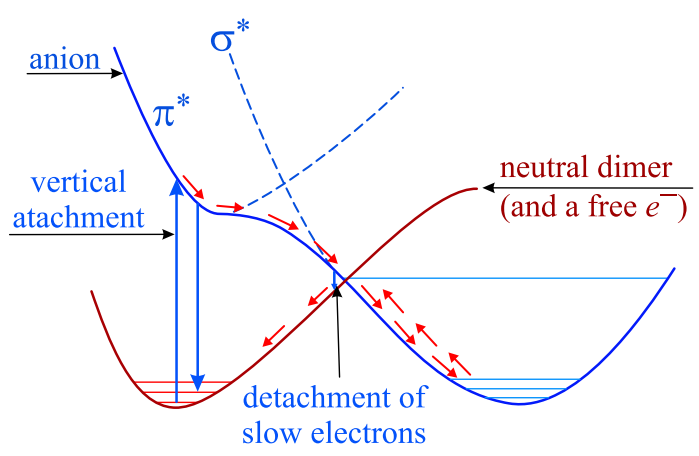

FIG. 5 (color online). Hypothetical potential curves of the formic acid dimer and monomer along the reaction coordinate of the electron-driven proton transfer.

The system may either lose a slow electron at the crossing of the anionic and neutral surfaces or proceed toward the minimum, consisting of the hydrogen-bonded complex of a formate anion and a dihydroxymethyl radical. This complex has enough vibrational energy to statistically return back to the crossing point, where a detachment of a slow electron may occur again. This mechanism thus represents a source of slow electrons and is capable of rationalizing the experimental observation reported in this work. The proton transfer is particularly efficient in competing with the very fast autodetachment, because it proceeds, like many acid-base reactions, without an activation barrier and because the low mass causes protons to move rapidly. It must be borne in mind, however, that the dynamics of electron collisions is complex and other factors, such as the modification of the resonance parameters upon hydrogen bonding, may also play a role.

The process observed here may be relevant for interactions of electrons with condensed matter, in particular, with biological materials having ubiquitous hydrogen bonds. It will have the consequence of inducing proton transfers and of rapidly slowing down electrons.

Finally, note that there is an analogy between the process proposed here and the ultrafast electron-driven proton transfer shown to enhance the photostability of proteins and other biomolecules by Sobolewski and Domcke (Refs. [25,26], and references therein). The present process is also ultrafast, as proven by the fact that it successfully competes with the very fast autodetachment in the early stages of the collision. In both cases, similar $\pi^{*}$ and $\sigma^{*}$ orbitals are temporarily singly occupied and drive the proton transfer. The systems differ in the overall charge; however, the present resonances do not have a "hole" in a valence orbital and are coupled to a continuum of free electrons.

This research is part of Project No. 200020-113599/1 of the Swiss National Science Foundation.
[1] B. Boudaiffa, P. Cloutier, D. Hunting, M. A. Huels, and L. Sanche, Science 287, 1658 (2000).

[2] F. Martin, P. D. Burrow, Z. Cai, P. Cloutier, D. Hunting, and L. Sanche, Phys. Rev. Lett. 93, 068101 (2004).

[3] S. Ptasinska, S. Denifl, V. Grill, T. D. Märk, E. Illenberger, and P. Scheier, Phys. Rev. Lett. 95, 093201 (2005).

[4] I. Bald, J. Kopyra, and E. Illenberger, Angew. Chem., Int. Ed. 45, 4851 (2006).

[5] M. Allan, J. Phys. B 39, 2939 (2006).

[6] I. Martin, T. Skalický, J. Langer, H. Abdoul-Carime, G. Karwasz, E. Illenberger, M. Stano, and S. Matejcik, Phys. Chem. Chem. Phys. 7, 2212 (2005).

[7] T. Sedlacko, R. Balog, A. Lafosse, M. Stano, S. Matejcik, R. Azria, and E. Illenberger, Phys. Chem. Chem. Phys. 7, 1277 (2005).

[8] F. A. Gianturco, R. R. Lucchese, J. Langer, I. Martin, M. Stano, G. Karwasz, and E. Illenberger, Eur. Phys. J. D 35, 417 (2005).

[9] I. Dabkowska, J. Rak, M. Gutowski, J. M. Nilles, S. T. Stokes, and K.H. Bowen, J. Chem. Phys. 120, 6064 (2004).

[10] I. Dabkowska, J. Rak, M. Gutowski, J. M. Nilles, S. T. Stokes, D. Radisic, and K. H. Bowen, Phys. Chem. Chem. Phys. 6, 4351 (2004).

[11] R. A. Bachorz, M. Haranczyk, I. Dabkowska, J. Rak, and M. Gutowski, J. Chem. Phys. 122, 204304 (2005).

[12] A. Pelc, W. Sailer, P. Scheier, N. J. Mason, E. Illenberger, and T. D. Märk, Vacuum 70, 429 (2003).

[13] T. N. Rescigno, C. S. Trevisan, and A. E. Orel, Phys. Rev. Lett. 96, 213201 (2006).

[14] V. Vizcaino, M. Jelisavcic, J. P. Sullivan, and S. J. Buckman, New J. Phys. 8, 85 (2006).

[15] F. A. Gianturco and R. R. Lucchese, Eur. Phys. J. D 39, 399 (2006).

[16] D. Ambrose and N. B. Ghiassee, J. Chem. Thermodyn. 19, 505 (1987).

[17] A. S. Coolidge, J. Am. Chem. Soc. 50, 2166 (1928).

[18] M. Allan, J. Phys. B 28, 5163 (1995).

[19] Y.-T. Chang, Y. Yamaguchi, W. H. Miller, and H.F. Schaefer III, J. Am. Chem. Soc. 109, 7245 (1987).

[20] G. M. Florio, T. S. Zwier, E. M. Myshakin, K. D. Jordan, and E. L. Sibert III, J. Chem. Phys. 118, 1735 (2003).

[21] M. Tronc, M. Allan, and F. Edard, in Proceedings of the 15th International Conference on Photonic, Electronic and Atomic Collisions, Brighton, England, 1987, Abstracts of Contributed Papers, edited by J. Geddes et al. (North-Holland, Amsterdam, 1987), p. 335.

[22] A. Aflatooni, B. Hitt, G. A. Gallup, and P.D. Burrow, J. Chem. Phys. 115, 6489 (2001).

[23] H. Hotop, M.-W. Ruf, M. Allan, and I. I. Fabrikant, Adv. At. Mol. Opt. Phys. 49, 85 (2003).

[24] M. Allan, J. Electron Spectrosc. Relat. Phenom. 48, 219 (1989).

[25] A. L. Sobolewski and W. Domcke, Chem. Phys. Chem. 7, 561 (2006).

[26] A. L. Sobolewski, W. Domcke, C. Dedonder-Lardeux, and C. Jouvet, Phys. Chem. Chem. Phys. 4, 1093 (2002). 